\title{
Empirical Study on the Influencing Factors of Financial Crisis Based on Cox Model--Taking the Data of Listed Companies in Overcapacity Industry as an Example \\ Kangru Liu Liang Dai
}

Guizhou University of Finance and Economics, Guiyang 550025, China

\begin{abstract}
This article adopts Cox Survival model to carry on the research of financial crisis early-warning, chooses the seven industries with surplus capacity in our country, and makes empirical research on the data of 900 listed companies of steel, coal, nonferrous metals, power generation, machinery and equipment, chemical industry and real estate, and 41 of the 900 listed companies are labeled In view of the 41 listed companies with the financial crisis, this paper chooses $70 \%$ as training samples, including 28 listed companies marked St, the remaining 30\% financial crisis listed companies as test samples, and predicts the forecast samples on the basis of training sample model. The factors that affect the financial crisis of enterprises are obtained. Cox model can be a comprehensive analysis of the factors influencing the financial crisis of listed companies.
\end{abstract}

\section{Keywords}

Financial crisis; Cox survival model; Excess capacity industry; Training samples; Test samples

\section{基于 Cox 模型的财务危机影响因素实证 研究一以产能过剩行业上市公司数据为例}

\author{
刘康儒 戴亮 \\ 贵州财经大学，贵阳 550025, 中国
}

摘要：本文采取 Cox 生存模型进行财务危机预警研究, 选取我国产能过剩七大行业, 钢铁、 煤炭、有色金属、电力发电、机械设备、化工、房地产 900 家上市公司数据为例进行实证研 究, 这 900 家上市公司中共有 41 家被标注 ST。针对产能过剩行业 41 家出现财务危机的上市 公司, 本文选取 $70 \%$ 作为训练样本, 包含 28 家被标注 ST 的上市公司, 剩下 $30 \%$ 出现财务危 机的上市公司为测试样本, 在训练样本的模型基础上对预测样本进行预测, 得出企业出现财 务危机的影响因素。Cox 模型能对上市公司财务危机影响因素全面分析。

关键词: 财务危机; Cox 生存模型; 产能过剩行业; 训练样本; 测试样本 


\section{1. 引言}

伊査克・爱迪思（2004）在其著作 《企业生命周期》中说道: “有生命的 有机体一一植物、动物和人类——都会 生长、长大、衰老和死亡, 其实组织也 一样。”从企业生存的自身因素来看, 根据国际四大会计事务所之一的普华 永道会计事务所在《2011 年中国企业长 期激励调研报告》这篇调查报告中提供 的数据, 我们可以得出各国企业的生命 周期普遍持续时间不长久的结论。其中, 我国中小企业的平均寿命仅为 2.5 年, 集团企业的平均寿命仅 7 至 8 年。相比 之下, 日本企业平均具有 12.5 年的寿命 而欧美企业平均具有 40 年的寿命。从 全球范围来看, 各国企业不仅呈现出平 均生命周期都不长的特征, 而且呈现出 高死亡、短寿命的态势。尤其在日益激 烈的市场竞争及瞬息万变的复杂环境 下，企业之间的竞争呈现白热化趋势， 时刻面临 “适者生存，优胜劣汰的命运 洗礼”, 许多企业一刻辉煌后瞬间败落, 或是停滞不前。可见, 增强企业的持续 经营能力从而延长其生存时间已经成 为了亟待推进的课题，而财务危机的爆 发则是影响企业生存时间的一个重要 因素。受 2008 年美国次贷危机影响, 不仅许多大型企业倒闭或破产清算, 众 多中小型企业与投资人更被卷入了倒 闭、亏损的游祸，使企业和社会蒙受巨 大损失。那么, 企业如何在危机丛林中 生存与发展呢? 这个问题让政府、社会 与企业都觉得需要共同面对、亟待解决。 美国著名管理学家斯蒂文. 芬克 (Steven Fink）在其《危机管理》一书中说: “危 机对企业, 对人类就像死亡和纳税一样 难以避免, 只有做好应对危机的准备和 计划, 才有力量与命运周旋。”依据企 业财务状况具有持续性和累积效应的 特点以及财务危机的发生是一个财务 状况由健康运营到财务风险、财务困境、
财务危机, 直到破产清算的逐步演化的 过程，应对企业不同周期的财务运营过 程进行跟踪、监控, 及早发现財务危机 信号, 因此寻找出哪些财务指标对企业 的持续运营具有保护作用, 哪些财务指 标不利于企业持续经营, 通过财务指标 来预测企业的财务危机, 使企业能够及 时应变, 延长企业生存时间, 提高企业 的持续经营能力，对于投资者、债权人、 政府或其他利益相关方来说, 可以减少 系统性风险发生的可能性。针对我国的 实际情况，在应对 2008 年国际金融危 机时我国采取了 4 万亿投资计划, 其引 致的产能持续释放, 使我国工业部门的 生产能力进一步扩大，一些工业部门相 继出现了产能过剩的问题。产能过剩导 致企业效益下降、甚至出现持续亏损, 这样会导致企业大面积倒闭破产，不仅 会诱发金融层面的系统性风险，还会加 剧经济社会环境的不稳定性。李克强总 理在 2016 年 3 月 5 日人民大会堂作政 府工作报告中再次强调, 要完成好三去 一降一补五大任务。财务危机预警在 “去杜杆 " 任务中为化解财务风险和 金融风险注入了重要的专业效能。因此 本文采用的数据为产能过剩行业上市 公司的数据。本文利用 Cox 生存模型是 半参数模型的特点, 采取 Cox 生存模型 对公司财务危机进行预测并分析哪些 财务指标是有利于企业的生存, 哪些企 业不利于企业的生存, 这样企业的管理 层和治理层发现这些财务指标异常时 可以及早引起重视, 寻找原因, 合理规 避财务危机。

\section{2. 理论模型介绍}

Cox 模型（又称 Cox 回归、比例风 险模型) 是一种最常用的生存数据分析 方法。其优点是适用范围很广以及便于 做多因素分析。危险函数和生存函数是 Cox 回归的核心概念。Cox 回归假定风 险函数如公式所示: $h(t)=$ 
$\mathrm{h}_{0}(\mathrm{t}) \exp \left(\mathrm{b}_{1} \mathrm{X}_{1}+\mathrm{b}_{2} \mathrm{X}_{2}+\cdots+\mathrm{b}_{\mathrm{p}} \mathrm{X}_{\mathrm{p}}\right)$ 其 中 $\mathrm{h}(\mathrm{t})$ 风险函数, 又称风险率或瞬间死 亡率, $\mathrm{h}_{0}(\mathrm{t})$ 是基准风险函数, 只相依于 时间, 不得为负值。因此, $h_{0}(t)$ 是一 个非特定的函数, 由于这个特性 Cox 回 归也被称为半参数模型, 不受协变量影 响。 $\mathrm{h}_{0}(\mathrm{t})$ 估计出发生目标事件的风险, 单位为 $\mathrm{t} ; \exp$ 是指数函数, $\mathrm{b}_{1} * \mathrm{X}_{1}+$ $\cdots+b_{p} * X_{p}$ 是时间不相依协变量 $X_{1}$, $\cdots, X_{p}$ 的线性函数, 将这个函数变为指数 函数, 以避免出现负值而无法处理; $\mathrm{X}$ 表示 $\mathrm{i}$ 个协变量的集束（“向量”)， 也就是对风险率可能产生影响的因素; $b_{1}, \cdots, b_{p}$ 表示将要估计的的协变量回归 系数。

\section{3. 样本和预警指标的选取}

\section{1. 样本的选取}

研究公司财务危机不得不考虑中 国资本市场的特殊性，在现有的绝大多 数文献中中国大陆的学者们把上市公 司被标注 ST 作为企业陷入财务危机的 标志, 考虑到这种情况本文亦参照这一 标准。这篇文章中 2014 年 1 月至 2017 年 7 月两年七个月时间为研究观测期, 选取沪深 A 股产能过剩的七大行业中 900 家上市公司进行研究。其中, 完全 数据样本为三年期间内被 ST 的公司, 其生存时间为公司上市到其发生 ST 的时间长度; 右删失数据为截止到 2017 年仍未被 ST 的公司, 成为非 ST 公 司，其生存时间为上市到 2017 年的时 间长度。数据来源 Wind 数据库。在剔 除相关要求不达标或者数据缺失的上 市公司后，此篇论文选取 2014 年 1 月 至 2017 年 7 月沪深 A 股产能过剩行业 900 家上市公司作为研究对象, 其中 ST 公司共 41 家, 非 ST 公司 859 家, 从 ST 公司随机选取 $70 \%$ 的公司即 28 家 ST 企业, 同时按照等比例原则选取 28 家 非 ST 上市公司作为训练样本, 其余
$30 \%$ 数据作为预测样本。因为有八家 ST 公司数据缺失, 所以最后选取 20 家 ST 公司和 20 家非 ST 公司作为训练样 本。

\section{2. 预警指标的选取}

根据 “杜邦分析法” 在这篇文章中 共选取了涵盖偿债、盈利、营运、现金 流量反映企业经营能力和财务状况的 24 个指标。考虑到在做最终的预警时的 协变量不需要太多, 所以我们要进行指 标篮选。第一步, 做财务指标非参数检 验: 用 SPSS 软件对 $\mathrm{t}-3$ 年的财务指标 进行显著性检验, 目的在于发现被标注 ST 上市公司与未被标注 ST 上市公司财 务指标上是否存在显著性差异, 并剔除 不存在显著性差异的指标。采用 SPSS23.0 中的 Mann-Whitney U 非参数 法对 ST 和非 ST 公司在发生财务危机的 t-3 年的财务数据进行检验,检验结果表 明: t-3 年 24 个财务指标中 12 个财务指 标通过了 Mann-Whitney U 检验的显著 性水平,充分说明这些指标在 ST 公司和 非 ST 公司中差别非常大,具有较高的判 别能力,这 12 个指标均能反映 $\mathrm{ST}$ 公司 和非 ST 公司之间的差异,适合作为上市 公司财务危机影响因素指标。

\section{4. 实证结果与分析}

\section{1. 建立模型}

此篇文章采用统计软件 SPSS23.0 的生存函数 Cox 回归进行建模, 在前 面部分通过因子分析我们已经找寻影 响企业财务困境的变量, 通过 Cox 回 归我们可以找到估计协变量系数和基 准风险函数。Cox 模型逐步回归的参数 估计结果见表 1 。 
表 1 训练样本实验结果

\begin{tabular}{|c|c|c|c|}
\hline & $\mathrm{B}$ & 标准误差 & 显著性 \\
\hline $\begin{array}{c}\text { 销售现金比 } \\
\text { 率 }\end{array}$ & -.041 & .023 & .076 \\
\hline $\begin{array}{c}\text { 全部资产现 } \\
\text { 金回收率 }\end{array}$ & -.097 & .115 & .399 \\
\hline $\begin{array}{c}\text { 流动比率 } \\
\text { 已获利息倍 } \\
\text { 数 }\end{array}$ & -2.010 & 1.087 & .064 \\
\hline $\begin{array}{c}\text { 现金流动负 } \\
\text { 债比 }\end{array}$ & -11.253 & 13.837 & .416 \\
\hline $\begin{array}{c}\text { 现金债务总 } \\
\text { 额比 }\end{array}$ & 20.514 & 11.614 & .077 \\
\hline $\begin{array}{c}\text { 总资产报酬 } \\
\text { 率 }\end{array}$ & -.183 & .415 & .659 \\
\hline $\begin{array}{c}\text { 成本费用利 } \\
\text { 润率 }\end{array}$ & .021 & .076 & .782 \\
\hline $\begin{array}{c}\text { 净资产收益 } \\
\text { 率 }\end{array}$ & .450 & .241 & .062 \\
\hline 每股收益 & -8.747 & 4.301 & .042 \\
\hline $\begin{array}{c}\text { 总资产净利 } \\
\text { 率 }\end{array}$ & -.654 & .648 & .312 \\
\hline 股东权益 & .076 & .037 & .037 \\
\hline
\end{tabular}
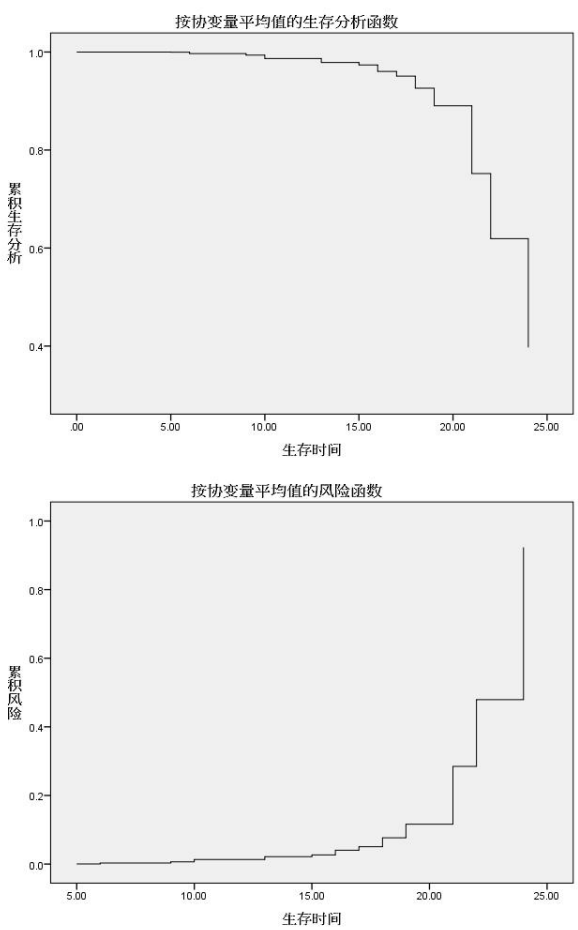

在训练样本的实验中, 我们以 0.1 为显著性指标，发现销售现金比率、流 动比率、现金债务总额比、净资产收益 率、每股收益、股东权益这些财务指标 显著。其中销售现金比率、流动比率、 每股收益的回归系数分别为 -0.041 、 $-2.010 、-8.747$, 系数为负表示该指标与 企业生存时间呈反向变动, 这些指标值 变大时企业的管理层和治理层要高度 重视、警惕，尤其是每股收益; 现金债 务总额比、净资产收益率、股东权益这 些指标的回归系数分别为 $20.514 、 0.450$ 、 0.076, 系数为正表示这些指标与企业生 存时间呈正方向变动, 有利于延长企业 生存时间, 尤其是现金负债总额比。从 生存函数图看, 模型预测训练样本的生 存时间不超过 25 年, 与实际结果一致。 针对训练样本进行实验后, 我们再对测 试样本进行实验:

\begin{tabular}{|c|c|c|c|}
\hline & B & 标准误差 & 显著性 \\
\hline $\begin{array}{c}\text { 销售现金 } \\
\text { 比率 }\end{array}$ & -.348 & .275 & .205 \\
\hline $\begin{array}{c}\text { 全部资产 } \\
\text { 现金回收 } \\
\text { 率 }\end{array}$ & 6.957 & 3.810 & .068 \\
\hline 流动比率 & -10.065 & 7. 120 & .157 \\
\hline $\begin{array}{c}\text { 已获利息 } \\
\text { 倍数 }\end{array}$ & -.981 & .533 & .066 \\
\hline $\begin{array}{c}\text { 现金流动 } \\
\text { 负债比 }\end{array}$ & 382.627 & 234.556 & .103 \\
\hline $\begin{array}{c}\text { 现金债务 } \\
\text { 总额比 }\end{array}$ & -937.766 & 523.070 & .073 \\
\hline $\begin{array}{c}\text { 总资产报 } \\
\text { 酬率 }\end{array}$ & -11.616 & 6.347 & .067 \\
\hline
\end{tabular}

续表 2 测试样本实验结果

\begin{tabular}{|c|c|c|c|}
\hline & B & 标准误差 & 显著性 \\
\hline $\begin{array}{c}\text { 成本费用 } \\
\text { 利润率 }\end{array}$ & .566 & .783 & .470 \\
\hline $\begin{array}{c}\text { 净资产收 } \\
\text { 益率 }\end{array}$ & -. 102 & .124 & .410 \\
\hline 每股收益 & -88.163 & 46. 176 & .056 \\
\hline $\begin{array}{c}\text { 总资产净 } \\
\text { 利率 }\end{array}$ & 15. 908 & 9.200 & .084 \\
\hline 股东权益 & -.248 & . 122 & .042 \\
\hline
\end{tabular}



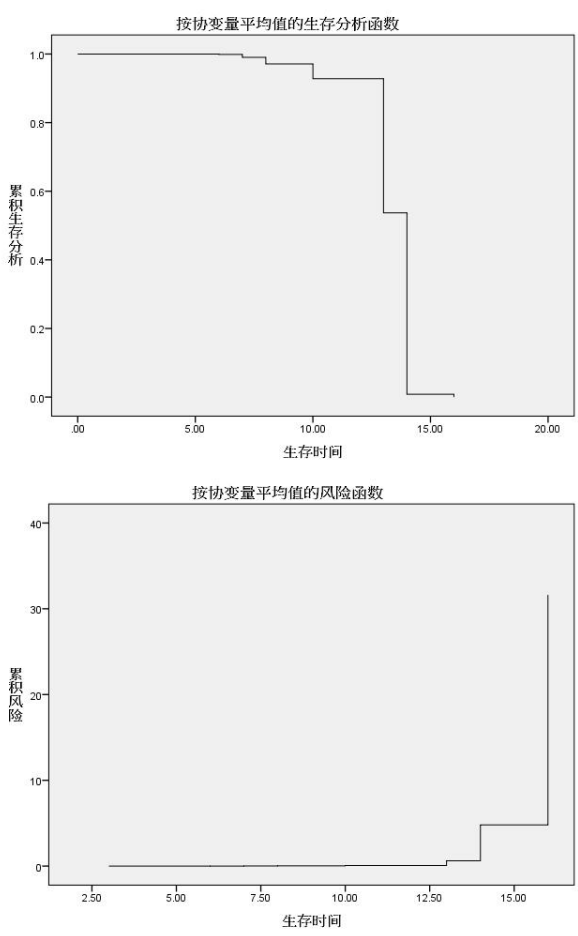

由测试样本实验结果来看, 与训练 样本的结果差异不大, 例如在每股收益 上都是呈负相关。从生存函数和风险函 数图来看, 模型预测测试样本的生存时 间不超过 16 年, 与实际结果基本一致, 说明 Cox 模型在预测企业生存时间上 效果整体不错。

\section{5. 结论}

此篇文章在结合了国内外众多学 者的研究基础之上将 Cox 生存函数模 型的理念引入上市公司风险预警中, 虽 然理论上用截面数据和时间序列进行 趋势预测 Cox 模型的优势。于此同时 利用训练样本建立模型, 利用预测样本 进行预测, 具有较强外推性和稳定性, Cox 模型还具有可以直接处理删失和截 尾数据样本, 无需样本配对的优势, 导 致其预测企业生存时间精度较高。从实 证得结果上来看, 在保障数据的真实性
时实证结果能够有效的找出影响企业 生存时间的财务指标, 此外, 对于采用 被 ST 作为上市公司出现财务困境的标 准, 虽然在理论界具有普遍性和适用性, 但是我国上市公司经营在经营期间企 业本身可能多次出现被 ST 和* ST 以 及 “摘帽” 和 “摘星” 的情况, 同时 由于相关法律法规还不完善导致借壳 上市还有不足之处, 可能一家大型经营 良好公司利用 ST 公司的 “壳资源” 造 成生存时间较短的现象，不符合 Cox 生存模型预测的条件, 对模型具有限制 性。

\section{参考文献}

[1] Beaver. W. Financial $R$ atios as Predictors of Failure $[\mathrm{J}]$.

Supplement to Journal of Accounting $R$ esearch, 1966, 32:71 - 111 .

[2] 韩国高、高铁梅、王立国、齐鹰飞、 王晓姝: 《中国制造业产能过剩的 测度、波动及成因研究》，《经济 研究》, 2011 年第 12 期

[3] 马超群, 何文. 基于 Cox 的财务困 境时点预测模型研究 $[\mathrm{J}]$. 统计与 决策，2010，21：38-42.

[4] Odom, M. D, R. Sharda. A

Neural Network Model for Bankruptcy in Proceedings of the International Joint $[\mathrm{J}]$. Conference on Neural Prediction. Networks, 1990(6) : $163-168$.

[5] 刘腾.股东层治理风险与公司绩效的 相关性研究 [J]. 财经界, 2007（1）.

[6] 李帆, 杜志涛, 李跨娟. 企业财务 预馨模型：理论回顾及其评论 [ J ] . 管理评论, 2011 [09]: 144-151.

[7] 倪中新，张杨. 基于 Cox 比例风险 模型的制造业财务困境恢复研究

$[\mathrm{J}]$. 统计与信息论坛, 2012, 27 (1) : 15-20. 
[8] 李维安, 谢永珍. 上市公司治理风险 预警指标体系的理论分析与实际验 证 [C]. 第四届南开大学公司治理 国际研讨会论文集，2007.

[9] 林毅夫、巫和禁、邢亦青: 《“潮 涌现象”与产能过剩的形成机制》, 《经济研究》，2010 年第 10 期。 